2017, Vol. 17, No. 1

ISSN $1667-4545$

Recuperado de www.revistas.unc.edu.ar

Laboratorio de Evaluación Psicológica y Educativa Facultad de Psicología - Universidad Nacional de Córdoba

\title{
Validación de la Escala Abreviada de Afrontamiento Religioso Brief-RCOPE en el Contexto Argentino en estudiantes de confesión católica
}

\author{
Joaquín Mezzadra* 1 , Hugo Simkin *2 \\ 1. UBACyT, Universidad de Buenos Aires, Argentina. \\ 2. Universidad de Buenos Aires, Argentina.
}

Introducción

Método

Resultados

Discusión

Referencias

Recibido: 02/11/2016 Revisado: 05/12/2016 Aceptado: 18/12/2016

\section{Resumen}

En la actualidad, el estudio del afrontamiento religioso constituye una de las principales líneas de investigación en psicología de la religión. Debido a la escasez de instrumentos que posibiliten su indagación en el contexto argentino, el presente estudio se propone adaptar y validar la Escala Abreviada de Afrontamiento Religioso (B-RCOPE), una de las técnicas más empleadas para su evaluación empírica. Participaron en el presente estudio 200 estudiantes de ambos sexos de colegios secundarios de confesión católica de la Ciudad Autónoma de Buenos Aires (CABA). De acuerdo con los resultados, el análisis factorial confirmatorio presenta un ajuste adecuado a los datos recabados de la población en estudio, de manera similar a lo reportado en otros trabajos. Por su parte, la consistencia interna resultó aceptable tanto para el afrontamiento positivo como para el afrontamiento negativo. De esta manera, los resultados obtenidos indican que la B-RCOPE cuenta con características psicométricas adecuadas, ofreciendo un aporte a la adaptación y la validación de este instrumento para el contexto argentino.

Palabras clave: afrontamiento religioso, afrontamiento, religiosidad, evaluación

\begin{abstract}
Currently, the study of religious coping is one of the main lines of research in psychology of religion. Since there is a lack of instruments in the Argentinean context, this study aims to adapt and validate the Brief Religious Coping Scale (B-RCOPE), one of the most widely used techniques for empirical evaluation. 200 students of both genders from secondary catholic schools in the Autonomous City of Buenos Aires participated in this study. According to our results, confirmatory factor analysis presents an adequate fit to the data, similar to those reported in other studies. Internal consistency was acceptable for both positive and negative coping. Results indicate that B-RCOPE has adequate psychometric characteristics for use within an Argentinean context.
\end{abstract}

Keywords: religious coping, coping, religiosity, measurement 


\section{Introducción}

Durante los últimos veinte años el número de estudios que centran su atención en el papel que ocupa la religión en el afrontamiento de estresores vitales ha crecido exponencialmente (Ano \& Vasconcelles, 2005; Pargament, 1997; Phelps et al., 2009). Diferentes autores han observado que algunas personas se apoyan en la religión como un recurso para comprender y afrontar momentos difíciles (Abu-Raiya \& Pargament, 2015; Ahles, Mezulis, \& Hudson, 2016; Feder et al., 2013). De acuerdo con Pargament (1997), el afrontamiento religioso puede definirse como un proceso orientado y contextual que pone a la religión como el eje central en el desarrollo de estrategias de resolución de conflictos y problemas. A su vez, el autor identifica patrones positivos y negativos de afrontamiento religioso para distinguir dos orientaciones que prevalecen en el individuo al momento de recurrir a la religión como estrategia de afrontamiento: mientras que los patrones positivos reflejan una relación segura con una fuerza trascendente, un sentido de conexión espiritual con los demás, y una visión benevolente del mundo; los negativos, por el contrario, reflejan tensiones espirituales subyacentes, descontento espiritual y luchas dentro de uno mismo, con los demás y con lo divino. Diversos estudios indican que las personas que viven su religión de manera positiva, generando vínculos de confianza con Dios, tienden a sobreponerse a diversos estresores con mayor facilidad que aquellas que experimentan una lucha interna constante con Dios o con su comunidad religiosa (Bryant-Davis et al., 2015; Lee, Nezu, \& Nezu, 2014; Rosmarin, Bigda-Peyton, Öngur, Pargament, \& Björgvinsson, 2013; Vallurupalli et al., 2012). De esta manera, el afrontamiento religioso ha sido vinculado a numerosas variables relativas, por ejemplo, estrés postraumático (Bryant-Davis \& Wong, 2013; Gerber, Boals, \& Schuettler, 2011; Leaman \& Gee, 2012), síntomas de ansiedad (Pirutinsky, Rosmarin, Pargament, \& Midlarsky, 2011), síntomas de depresión (Carpenter, Laney, \& Mezulis, 2012), duelo (Ke1ley \& Chan, 2012; Lee, Roberts, \& Gibbons, 2013), o enfermedades médicas (Maciejewski et al., 2012; Rand et al., 2012; Thuné-Boyle, Stygall, Keshtgar, Davidson, \& Newman, 2013; Trevino, Archambault, Schuster, Richardson, \& Moye, 2012).

Cabe señalar que en la literatura especializada suelen distinguirse los términos espiritualidad y religiosidad. De esta manera, mientras que la espiritualidad es considerada como una motivación que orienta el comportamiento en el esfuerzo de construir un sentido más profundo para la vida desde una perspectiva escatológica (relativa al destino del ser humano luego de su muerte), la religiosidad remite al modo en que la espiritualidad es moldeada y se expresa a través de una organización comunitaria o social (Piedmont, 2012).

Para la evaluación del afrontamiento religioso, Pargament y colegas (1988) construyeron la Escala de Afrontamiento Religioso (en adelante, RCOPE), compuesta inicialmente por 105 ítems agrupados en dos factores: (1) afrontamiento religioso positivo y (2) afrontamiento religioso negativo. La redacción de los ítems derivó de una exhaustiva revisión de la literatura y de entrevistas con población religiosa. Posteriormente, la técnica fue puesta a prueba con dos muestras, la primera de 540 estudiantes universitarios que habían experimentado un grave acontecimiento negativo en sus vidas, y la segunda de 551 adultos hospitalizados que sufrían de enfermedades médicas (Pargament et al., 1988). Sin embargo, si bien las propiedades psicométricas de la técnica alcanzaron los guarismos o valores esperados (Pargament, Koenig, Tarakeshwar, \& Hahn, 2001, 2004), una de las principales limitaciones consistió en su excesiva longitud (Pargament, Feuille, \& Burdzy, 2011). Por este motivo, los autores construyeron la Brief-RCOPE Scale (en ade- 
lante, B-RCOPE), una versión abreviada de la escala que conserva la fundamentación teórica del instrumento original. La primera versión de la técnica incluyó 21 ítems y fue probada en una muestra de habitantes de localidades cercanas a la ciudad de Oklahoma, luego del atentado sufrido en 1995 (Pargament, Smith, Koenig, \& Perez, 1998). Posteriormente, empleando una muestra de estudiantes universitarios, se realizó un análisis factorial de la B-RCOPE limitado a dos dimensiones, cada una compuesta por 7 ítems, que identifican grupos de patrones o estrategias de afrontamiento religioso positivos $\mathrm{y}$ negativos (Pargament et al., 1998).

Si bien la mayoría de los estudios que emplean la B-RCOPE han sido llevados a cabo en Estados Unidos (Pargament et al., 2011), se han registrado numerosos desarrollos en diferentes contextos, como Alemania (Zwingmann, Müller, Körber, \& Murken, 2008; Zwingmann, Wirtz, Müller, Körber, \& Murken, 2006), Brasil (Freitas et al., 2015; Ramirez et al., 2012), Canadá (Houck, 2012), Corea (Noh, Chang, Jang, Lee, \& Lee, 2016), Croacia (Mihaljević, Aukst-Margetić, Vuksan-Ćusa, Koić, \& Milošević, 2012), España (Martinez \& Sousa, 2011), Francia (Caporossi, Trouillet, \& Brouillet, 2012), Grecia (Kapsou, Panayiotou, Kokkinos, \& Demetriou, 2010), Holanda (Braam et al., 2008, 2010), India (Grover, Sarkar, Bhalla, Chakrabarti, \& Avasthi, 2016; Roberts \& Montgomery, 2015), Inglaterra (Brewer, Robinson, Sumra, Tatsi, \& Gire, 2015; Thuné-Boyle et al., 2013), Italia (Giaquinto, Cipolla, Giachetti, \& Onorati, 2011), Irak (Al-Hadethe, Hunt, Thomas, \& Al-Qaysi, 2014), Irán (Gholamzadeh, Hamid, Basri, Sharif, \& Ibrahim, 2014), Malasia (Nurasikin et al., 2013), México (Acevedo-Alemán \& González-Tovar, 2014; Rivera-Ledesma \& Montero-López, 2007; Robles-García et al., 2014), Nigeria (Amadi, Uwakwe, Aguocha, et al., 2016; Amadi, Uwakwe, Ndukuba, et al., 2016), Nueva Zelanda (Gardner, Krägeloh, \& Henning, 2014), Pakistán (Khan \& Watson, 2006), Puer- to Rico (Rodríguez-Carrión, Sayers-Montalvo, \& Martínez-Taboas, 2011), Ruanda (Schaal, Heim, \& Elbert, 2014), Suiza (Fitchett, Winter-Pfandler, \& Pargament, 2014) y Tanzania (Watt et al., 2014). Los estudios que emplean la escala RCOPE han utilizado muestras de población adolescente y adulta, tanto religiosa como no religiosa, lo que contribuye a confirmar el modelo propuesto por el autor. Además, los resultados de estos trabajos han mostrado propiedades psicométricas aceptables en cuanto a la consistencia interna del instrumento $(\alpha>.70)$. Adicionalmente, en la mayoría de los estudios confirmatorios relevados los valores de los índices de ajuste (IFI, NNFI y RMSEA) alcanzaron los guarismos esperados.

Finalmente, cabe señalar que si bien existen antecedentes de la escala en el contexto latinoamericano, por ejemplo en México, Puerto Rico y Brasil, aún resulta necesario incrementar la cantidad de estudios que contribuyan a contar con instrumentos válidos y confiables para su administración en el contexto local. Por este motivo, el presente trabajo se propone realizar una adaptación de la técnica al contexto argentino.

\section{Método}

\section{Participantes}

Se trabajó con un muestreo no probabilístico incidental en el que participaron 200 adolescentes con edades entre 14 y 18 años $(\mathrm{M}=$ 15.87; $\mathrm{DE}=1.11$ ) y de ambos sexos (Hombres $=46.4 \%$; Mujeres $=54.6 \%$ ), que cursaban sus estudios secundarios en un colegio privado de confesión católica en la Ciudad Autónoma de Buenos Aires (CABA). Para la selección de los participantes se tuvieron en cuenta las recomendaciones de Pargament, Koenig y Perez (2000), quienes sugieren como condición para formar parte del estudio que los individuos se consideren a sí mismos religiosos. 


\section{Instrumento}

Los datos fueron recolectados a través de un instrumento de evaluación autoadministrado, asegurando el anonimato de los participantes. El mismo estuvo compuesto por:

\section{Escala Abreviada de Afrontamiento Religioso} / Brief Measure of Religious Coping (B-RCOPE; Pargament et al., 1998). Para la evaluación del afrontamiento religioso se utilizó la Escala B-RCOPE, compuesta por 14 ítems que evalúan dos patrones de afrontamiento religioso en población adolescente y adulta: (1) Afrontamiento religioso positivo (positive religious coping; $\mathrm{p}$. ej., Trato de comprender que Dios me fortalece a través de ciertas situaciones; siete ítems: 2,4 , $5,6,7,10$ y 14) y (2) Afrontamiento religioso negativo (negative religious coping; p. ej., Me siento castigado por Dios por mi falta de devoción; siete ítems: 1, 3, 8, 9, 11, 12 y 13). Si bien en su versión original la escala presenta un formato de respuesta tipo Likert con cuatro anclajes de respuesta en función del grado de acuerdo de los participantes, entre 0 (De ningún modo) y 3 (Mucho) (Martinez \& Sousa, 2011; Pargament et al., 1998) o entre 0 (Nunca) y 3 (Siempre) (Ramirez et al., 2012); se ha preferido optar por cinco anclajes de respuesta, desde 1 (Nunca) hasta 5 (Siempre). La decisión se debe a que se prefiere una modalidad que permita consignar como respuesta "A veces", evitando de esta manera forzar una respuesta que se incline en alguna dirección (Nunca - Siempre). Puntuaciones mayores en cada dimensión indican mayores niveles de afrontamiento religioso positivo $\mathrm{y}$ de afrontamiento religioso negativo. Para el presente estudio se realizó una traducción de la versión original de la escala (Pargament et al., 1998), cuya consistencia interna resultó adecuada tanto para el afrontamiento religioso positivo (con valores alfa entre .90 y .87) como para el afrontamiento religioso negativo (con valores alfa entre .81 y .78), de acuerdo con la literatu- ra especializada (Martínez-Arias, Hernández, \& Lloreda, 2006).

Cuestionario de datos personales. Se elaboró un cuestionario ad hoc para relevar información sociodemográfica (p. ej., edad).

\section{Procedimiento}

En la adaptación de la escala B-RCOPE al contexto argentino se siguieron los estándares metodológicos internacionales recomendados por la International Test Commission (ITC) para una adaptación correcta del instrumento a diferentes contextos culturales (Muñiz, Elosua, \& Hambleton, 2013; Muñiz \& Hambleton, 2000). En primer lugar, se realizó una retrotraducción de la escala, respetando el contenido original de cada ítem y procurando reflejar el constructo original de cada dimensión. Así, la traducción no fue realizada de manera literal, sino que buscó conservar el sentido psicológico de cada ítem. Se contó con la participación de dos traductores no familiarizados con el instrumento. Luego de realizar la traducción, se compararon ambas versiones en inglés del instrumento. Se conservaron aquellos ítems que pudieron mantener el sentido psicológico del tema y se repitió el proceso hasta contar la totalidad de los ítems traducidos. Posteriormente, los estudiantes fueron invitados a participar de forma voluntaria en la investigación, solicitándoles el consentimiento informado e indicando que en cualquier momento podían oponerse a responder, siendo libres de abandonar el estudio si lo consideraban adecuado. Finalmente, se les hizo constar a los participantes que los datos derivados de esta investigación se utilizarían con fines exclusivamente científicos bajo la Ley Nacional 25.326 de protección de los datos personales (2000). 
Análisis de Datos

Con el objeto de adaptar la B-RCOPE al contexto local se emplearon medidas estadísticas descriptivas y modelos multivariantes. Con tal finalidad se trabajó con estadística descriptiva e inferencial mediante el software estadístico SPSS 21 (IBM Corporation, 2012) y Lisrel 8 (Scientific Software International, 2006). Se analizó la confiabilidad de la B-RCOPE a partir del análisis de consistencia interna, optando por emplear el estadístico alfa ordinal. En segundo lugar, se evaluó la validez de constructo empleando un análisis factorial confirmatorio (en adelante, AFC).

\section{Resultados}

Dada la naturaleza ordinal de los ítems, se decidió aplicar el análisis utilizando una matriz de correlaciones policóricas. Con el objeto de adaptar y validar la B-RCOPE en el contexto local, en primer lugar se realizó el AFC, evaluado a partir de los índices de bondad de ajuste $\chi^{2}$, IFI (incremental fit index), NNFI (non-normed fit index), CFI (comparative fit index) y SRMR (standardized root mean residual), siguiendo las recomendaciones de la literatura (Holgado-Tello, Chacón-Moscoso, Barbero-García, \& Vila-Abad, 2009; Schreiber, Nora, Stage, Barlow, \& King, 2006). Tal como puede observarse en la Tabla 1, el análisis ha permitido comprobar que el modelo propuesto se ajusta a los datos recabados en campo (Hair, Black, Babin, \& Anderson, 2009; Schreiber et al., 2006). Además, los índices IFI, NNFI y CFI alcanzaron valores superiores a 90 y el índice SRMR obtuvo valores inferiores a .1, guarismos que suelen aceptarse como indicadores de un ajuste razonable (Harrington, 2009; Kline, 2005; Schreiber et al., 2006).
Tabla 1

Análisis Factorial Confirmatorio de la escala B-RCOPE.

\begin{tabular}{cccccc}
\hline & $\mathbf{X}_{(\mathbf{g l})}$ & NNFI & CFI & IFI & SRMR \\
\hline RCOPE & $2996.906_{(91)}$ & .93 & .94 & .94 & .09 \\
\hline
\end{tabular}

Nota. Modelo bidimensional.

A partir de los resultados del AFC, puede observarse el modelo de la Escala de Afrontamiento Religioso en la Figura 1.

Posteriormente, se evaluó la consistencia interna a partir del estadístico alfa ordinal, tal como ha sido sugerido en la literatura (Elosua-Oliden \& Zumbo, 2008), tanto para el afrontamiento positivo $(\alpha=.83)$, como para el afrontamiento negativo $(\alpha=.72)$.

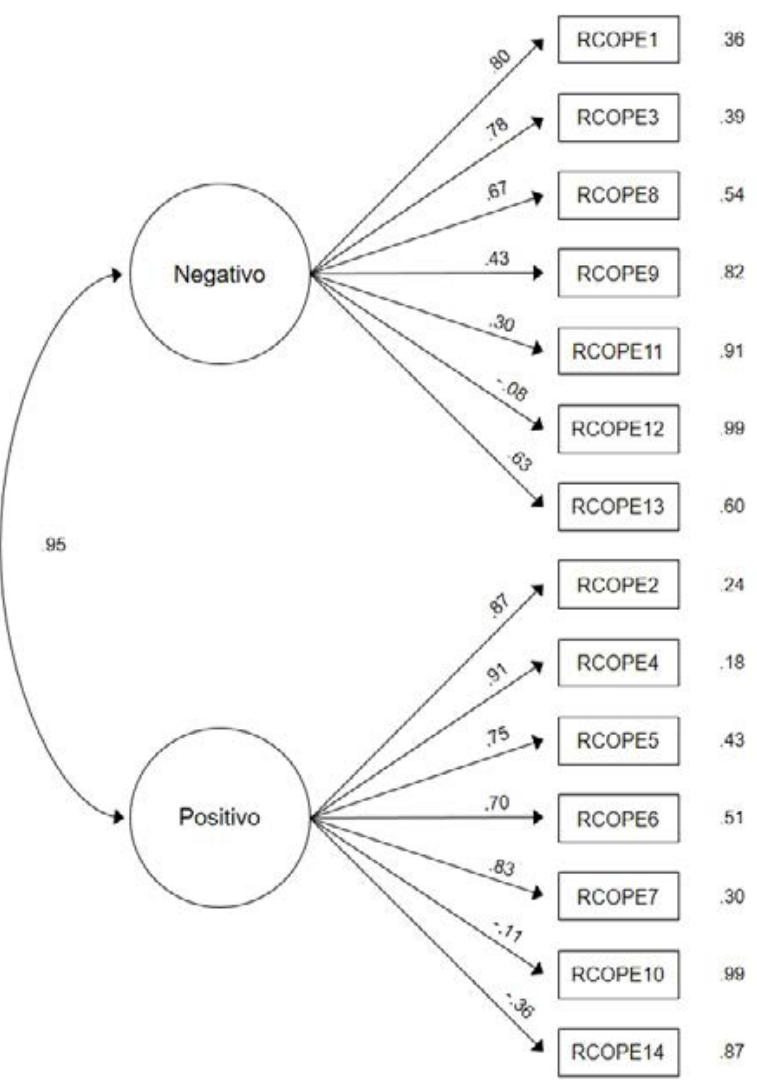

Figura 1.

Modelo estructural de la Escala Abreviada de Afrontamiento Religioso.

Nota. $* p<.05 ; * * p<.01$. 


\section{Discusión}

La escala B-RCOPE ha presentado propiedades psicométricas adecuadas para su empleo en el contexto local y sus ítems resultan similares a los propuestos en la versión original (Pargament et al., 1998). El AFC presenta un ajuste adecuado a los datos recabados en la población en estudio, de manera similar a lo reportado en los antecedentes (Pargament et al., 2011). Puede señalarse que, tal como se puede observar en la Figura 1, la covarianza superior a .65 podría contribuir a hipotetizar una estructura unidimensional (Lévy-Mangin \& Varela, 2006). Sin embargo, actualmente no se cuenta con apoyo teórico que pueda contribuir a sostener un modelo de tales características. En segundo lugar, se destaca que los parámetros estimados alcanzan guarismos adecuados, considerando que de acuerdo con la literatura, las cargas factoriales en psicología tienden a oscilar en torno a .50 (Beauducel \& Herzberg, 2006). Cabe señalar que si bien se observa la presencia de ecuaciones infractoras (parámetros estimados negativos), investigaciones en la temática han demostrado que los índices de ajuste no se ven significativamente afectados por la presencia de dichas ecuaciones infractoras (Chen, Bollen, Paxton, Curran, \& Kirby, 2001). Por esa razón se ha decidido conservar el modelo original.

En cuanto a la consistencia interna cabe destacar que se alcanzaron guarismos aceptables para cada factor $(\alpha>, 70)$, de acuerdo con la literatura (Freiberg-Hoffmann, Stover, de la Iglesia, \& Fernández-Liporace, 2013; Martinez-Arias, Hernández-Lloreda, \& Hernández-Lloreda, 2006).

En lo que respecta a las limitaciones del estudio, sería conveniente que futuros trabajos cuenten con muestras representativas. Asimismo, resulta necesario explorar las propiedades psicométricas de la escala en diferentes contextos religiosos, debido a que la mayoría de los estudios en el área emplean muestras de estu- diantes universitarios o de población religiosa cristiana (Hill, 2012; Kapuscinski \& Masters, 2010).

Finalmente, se señala que en los últimos años en diversos países se ha avanzado considerablemente en la adaptación y validación de la escala de 14 ítems B-RCOPE, por lo que el presente trabajo aporta resultados tentativos para su empleo en el contexto argentino. De esta manera, se espera poder aportar una medida válida y confiable para que futuros estudios puedan profundizar en la comprensión de las relaciones entre el afrontamiento religioso y otros constructos psicológicos.

\section{Referencias}

Abu-Raiya, H., \& Pargament, K. I. (2015). Religious coping among diverse religions: Commonalities and divergences. Psychology of Religion and Spirituality, 7(1), 24-33. doi: 10.1037/a0037652

Acevedo-Alemán, J., \& González-Tovar, J. (2014). No envejecemos igual: La religiosidad y el género en adultos mayores del noreste de México. Revista Reflexiones, 93(1), 133-144. Recuperado de: http:// revistas.ucr.ac.cr/index.php/reflexiones

Ahles, J. J., Mezulis, A. H., \& Hudson, M. R. (2016). Religious coping as a moderator of the relationship between stress and depressive symptoms. Psychology of Religion and Spirituality, 8(3), 228-234. doi: $10.1037 /$ rel0000039

Al-Hadethe, A., Hunt, N., Thomas, S., \& Al-Qaysi, A. (2014). Cross-cultural validation and psychometric properties of the Arabic Brief Religious Coping Scale (A-BRCS). Journal of Religion and Health, 55(1), 16-25. doi: 10.1007/s10943-014-9963-7

Amadi, K. U., Uwakwe, R., Aguocha, C. M., Ezeme, M. S., Muomah, R. C., Ndukuba, A. C., \& Odinka, P. C. (2016). Illness coping behaviour of outpatients with depression or diabetes mellitus in two tertiary hospitals in Enugu state, southeast Nigeria. Mental Health, Religion \& Culture, 19(4), 371-378. doi: 
10.1080/13674676.2016.1201055

Amadi, K. U., Uwakwe, R., Ndukuba, A. C., Odinka, P. C., Igwe, M. N., Obayi, N. K., \& Ezeme, M. S. (2016). Relationship between religiosity, religious coping and socio-demographic variables among out-patients with depression or diabetes mellitus in Enugu, Nigeria. African Health Sciences, 16(2), 497. doi: 10.4314/ahs.v16i2.18

Ano, G. G., \& Vasconcelles, E. B. (2005). Religious coping and psychological adjustment to stress: A meta-analysis. Journal of Clinical Psychology, 61(4), 461-480. doi: 10.1002/jclp.20049

Beauducel, A., \& Herzberg, P. Y. (2006). On the performance of maximum likelihood versus means and variance adjusted weighted least squares estimation in CFA. Structural Equation Modeling: A Multidisciplinary Journal, 13(2), 186-203. doi: $10.1207 / \mathrm{s} 15328007 \mathrm{sem} 1302 \_2$

Braam, A. W., Schaap-Jonker, H., Mooi, B., de Ritter, D., Beekman, A. T. F., \& Deeg, D. J. H. (2008). God image and mood in old age: Results from a community-based pilot study in the Netherlands. Mental Health, Religion \& Culture, 11(2), 221-237. doi: 10.1080/13674670701245274

Braam, A. W., Schrier, A. C., Tuinebreijer, W. C., Beekman, A. T. F., Dekker, J. J. M., \& de Wit, M. A. S. (2010). Religious coping and depression in multicultural Amsterdam: A comparison between native Dutch citizens and Turkish, Moroccan and Surinamese/Antillean migrants. Journal of Affective Disorders, 125(1-3), 269-278. doi: 10.1016/j. jad.2010.02.116

Brewer, G., Robinson, S., Sumra, A., Tatsi, E., \& Gire, N. (2015). The influence of religious coping and religious social support on health behaviour, health status and health attitudes in a British Christian sample. Journal of Religion and Health, 54(6), 2225-2234. doi: 10.1007/s10943-014-9966-4

Bryant-Davis, T., Ullman, S., Tsong, Y., Anderson, G., Counts, P., Tillman, S. ... Gray, A. (2015). Healing pathways: Longitudinal effects of religious coping and social support on PTSD symptoms in African American sexual assault survivors. Journal of Trauma \& Dissociation, 16(1), 114-128. doi:

\section{$10.1080 / 15299732.2014 .969468$}

Bryant-Davis, T., \& Wong, E. C. (2013). Faith to move mountains: Religious coping, spirituality, and interpersonal trauma recovery. American Psychologist, 68(8), 675-684. doi: 10.1037/a0034380

Caporossi, J., Trouillet, R., \& Brouillet, D. (2012). Validation de la version française d'une échelle abrégée de coping religieux: Brief-RCOPE. Psychologie Française, 1-15. doi: 10.1016/j.psfr.2011.12.001

Carpenter, T. P., Laney, T., \& Mezulis, A. (2012). Religious coping, stress, and depressive symptoms among adolescents: A prospective study. Psychology of Religion and Spirituality, 4(1), 19-30. doi: 10.1037/a0023155

Chen, F., Bollen, K., Paxton, P., Curran, P., \& Kirby, J. (2001). Improper solutions in structural equation models: Causes, consequences, and strategies. Sociological Methods \& Research, 29(4), 468-508. doi: 10.1177/0049124101029004003

Elosua-Oliden, P., \& Zumbo, B. D. (2008). Coeficientes de fiabilidad para escalas de respuesta categórica ordenada. Psicothema, 20(4), 896-901. Recuperado de http://www.psicothema.com

Feder, A., Ahmad, S., Lee, E. J., Morgan, J. E., Singh, R., Smith, B. W. ... Charney, D. S. (2013). Coping and PTSD symptoms in Pakistani earthquake survivors: Purpose in life, religious coping and social support. Journal of Affective Disorders, 147(1-3), 156-163. doi: 10.1016/j.jad.2012.10.027

Fitchett, G., Winter-Pfandler, U., \& Pargament, K. I. (2014). Struggle with the divine in Swiss patients visited by chaplains: Prevalence and correlates. Journal of Health Psychology, 19(8), 966-976. doi: $10.1177 / 1359105313482167$

Freiberg-Hoffmann, A., Stover, J. B., de la Iglesia, G., \& Fernández-Liporace, M. (2013). Correlaciones policóricas y tetracóricas en estudios factoriales exploratorios y confirmatorios. Ciencias Psicológicas, $7(2), 151-164$.

Freitas, T. H., Hyphantis, T. N., Andreoulakis, E., Quevedo, J., Miranda, H. L., Alves, G. S. ... Carvalho, A. F. (2015). Religious coping and its influence on psychological distress, medication adherence, and quality of life in inflammatory bowel disease. 
Revista Brasileira de Psiquiatria, 37(3), 219-227. doi: 10.1590/1516-4446-2014-1507

Gardner, T. M., Krägeloh, C. U., \& Henning, M. A. (2014). Religious coping, stress, and quality of life of Muslim university students in New Zealand. Mental Health, Religion \& Culture, 17(4), 327-338. doi: 10.1080/13674676.2013.804044

Gerber, M. M., Boals, A., \& Schuettler, D. (2011). The unique contributions of positive and negative religious coping to posttraumatic growth and PTSD. Psychology of Religion and Spirituality, 3(4), 298307. doi: $10.1037 / \mathrm{a} 0023016$

Gholamzadeh, S., Hamid, T. A., Basri, H., Sharif, F., \& Ibrahim, R. (2014). Religious coping and psychological well-being among Iranian stroke caregivers. Iranian Journal of Nursing and Midwifery Research, 19(5), 478-84. Recuperado de http://ijnmr.mui.ac.ir/index.php/ijnmr

Giaquinto, S., Cipolla, F., Giachetti, I., \& Onorati, D. (2011). Italian validation of the Brief RCOPE scale for religious coping. Journal of Medicine and the Person, 9(2), 70-75. doi: 10.1007/s12682-0110087-5

Grover, S., Sarkar, S., Bhalla, A., Chakrabarti, S., \& Avasthi, A. (2016). Religious coping among selfharm attempters brought to emergency setting in India. Asian Journal of Psychiatry, 23, 78-86. doi: 10.1016/j.ajp.2016.07.009

Hair, J. F., Black, W. C., Babin, B. J., \& Anderson, R. E. (2009). Multivariate Data Analysis. London: Prentice Hall.

Harrington, D. (2009). Confirmatory Factor Analysis. Oxford: Oxford University Press.

Hill, P. C. (2012). Measurement assessment and issues in the Psychology of Religion and Spirituality. En R. F. Paloutzian \& C. L. Park (Eds.), Handbook of Psychology of Religion and Spirituality (pp. 4875). New York: The Guilford Press.

Holgado-Tello, F. P., Chacón-Moscoso, S., Barbero-García, I., \& Vila-Abad, E. (2009). Polychoric versus Pearson correlations in exploratory and confirmatory factor analysis of ordinal variables. Quality and Quantity, 44(1), 153-166. doi: 10.1007/ s11135-008-9190-y

Houck, J. (2012). Finding a voice: Affirming religious coping as a strength among disenfranchised appalachians. Journal of Appalachian Studies, 18(1), 189-205. Recuperado de http://appalachianstudies. org/journal

IBM Corporation. (2012). IBM SPSS Statistics (21). Recuperado de http://www-01.ibm.com/software/es/ analytics/spss

Kapsou, M., Panayiotou, G., Kokkinos, C. M., \& Demetriou, A. G. (2010). Dimensionality of coping: An empirical contribution to the construct validation of the Brief-COPE with a Greek-speaking sample. Journal of Health Psychology, 15(2), 215-229. doi: 10.1177/1359105309346516

Kapuscinski, A. N., \& Masters, K. S. (2010). The current status of measures of spirituality: A critical review of scale development. Psychology of Religion and Spirituality, 2(4), 191-205. doi: 10.1037/a0020498

Kelley, M. M., \& Chan, K. T. (2012). Assessing the role of attachment to God, meaning, and religious coping as mediators in the grief experience. Death Studies, 36(3), 199-227. doi: 10.1080/07481187.2011.553317

Khan, Z. H., \& Watson, P. J. (2006). Construction of the Pakistani Religious Coping Practices Scale: Correlations with religious coping, religious orientation, and reactions to stress among Muslim university students. International Journal for the Psychology of Religion, 16(2), 101-112. doi: 10.1207/ s15327582ijpr1602_2

Kline, R. B. (2005). Structural equation modeling. New York: The Guilford Press.

Leaman, S. C., \& Gee, C. B. (2012). Religious coping and risk factors for psychological distress among African torture survivors. Psychological Trauma: Theory, Research, Practice, and Policy, 4(5), 457465. doi: 10.1037/a0026622

Lee, M., Nezu, A. M., \& Nezu, C. M. (2014). Positive and negative religious coping, depressive symptoms, and quality of life in people with HIV. Journal of Behavioral Medicine, 37(5), 921-930. doi: 10.1007/s10865-014-9552-y

Lee, S. A., Roberts, L. B., \& Gibbons, J. A. (2013). 
When religion makes grief worse: Negative religious coping as associated with maladaptive emotional responding patterns. Mental Health, Religion \& Culture, 16(3), 291-305. doi: 10.1080/13674676.2012.659242

Lévy-Mangin, J. P., \& Varela, J. (2006). Modelización con estructuras de covarianzas en ciencias sociales. España: Netbiblio.

Ley $\mathrm{N}^{\mathrm{o}}$ 25.326. 2000. Protección de datos personales. Argentina. Recuperado de: http://servicios.infoleg. gob.ar/infolegInternet

Maciejewski, P. K., Phelps, A. C., Kacel, E. L., Balboni, T. A., Balboni, M., Wright, A. A. ... Prigerson, H. G. (2012). Religious coping and behavioral disengagement: Opposing influences on advance care planning and receipt of intensive care near death. Psycho-Oncology, 21(7), 714-723. doi: 10.1002/ pon. 1967

Martinez, N. C., \& Sousa, V. D. (2011). Cross-cultural validation and psychometric evaluation of the Spanish Brief Religious Coping Scale (S-BRCS). Journal of Transcultural Nursing, 22(3), 248-256. doi: 10.1177/1043659611404426

Martinez-Arias, M. R., Hernández-Lloreda, M. J., \& Hernández-Lloreda, M. V. (2006). Psicometría. Madrid: Alianza Editorial.

Mihaljević, S., Aukst-Margetić, B., Vuksan-Ćusa, B., Koić, E., \& Milošević, M. (2012). Hopelessness, suicidality and religious coping in Croatian war veterans with PTSD. Psychiatria Danubina, 24(3), 292-297. Recuperado de http://www.hdbp.org/ psychiatria_danubina/index.html

Muñiz, J., Elosua, P., \& Hambleton, R. K. (2013). Directrices para la traducción y adaptación de los tests: Segunda edición. Psicothema, 25(2), 151-157. Recuperado de http://www.psicothema.com

Muñiz, J., \& Hambleton, R. K. (2000). Adaptación de los test de unas culturas a otras. Metodología de las Ciencias del Comportamiento, 2(2), 129-149. Recuperado de: https://dialnet.unirioja.es/servlet/ revista? codigo $=5628$

Noh, H., Chang, E., Jang, Y., Lee, J. H., \& Lee, S. M. (2016). Suppressor effects of positive and negative religious coping on academic burnout among Ko- rean middle school students. Journal of Religion and Health, 55(1), 135-146. doi: 10.1007/s10943015-0007-8

Nurasikin, M., Khatijah, L., Aini, A., Ramli, M., Aida, S., Zainal, N., \& Ng, C. (2013). Religiousness, religious coping methods and distress level among psychiatric patients in Malaysia. International Journal of Social Psychiatry, 59(4), 332-338. doi: $10.1177 / 0020764012437127$

Pargament, K. I. (1997). The Psychology of Religion and Coping: Theory, Research, Practice. New York: Guilford Press.

Pargament, K. I., Feuille, M., \& Burdzy, D. (2011). The Brief RCOPE: Current psychometric status of a short measure of religious coping. Religions, 2(4), 51-76. doi: 10.3390/rel2010051

Pargament, K. I., Kennell, J., Hathaway, W., Grevengoed, N., Newman, J., \& Jones, W. (1988). Religion and the problem-solving process: Three styles of coping. Journal for the Scientific Study of Religion, 27(1), 90. doi: 10.2307/1387404

Pargament, K. I., Koenig, H. G., \& Perez, L. M. (2000). The many methods of religious coping: Development and initial validation of the RCOPE. Journal of Clinical Psychology, 56(4), 519-543. doi: 10.1002/(SICI)1097-4679(200004)56:4<519::AI D-JCLP6>3.0.CO;2-1

Pargament, K. I., Koenig, H. G., Tarakeshwar, N., \& Hahn, J. (2001). Religious struggle as a predictor of mortality among medically ill elderly patients. Archives of Internal Medicine, 161(15), 18811885. doi: 10.1001/archinte.161.15.1881

Pargament, K. I., Koenig, H. G., Tarakeshwar, N., \& Hahn, J. (2004). Religious coping methods as predictors of psychological, physical and spiritual outcomes among medically ill elderly patients: A two-year longitudinal study. Journal of Health Psychology, 9(6), 713-730. doi: 10.1177/1359105304045366

Pargament, K. I., Smith, B. W., Koenig, H. G., \& Perez, L. (1998). Patterns of positive and negative religious coping with major life stressors. Journal for the Scientific Study of Religion, 37(4), 710. doi: $10.2307 / 1388152$

Phelps, A. C., Maciejewski, P. K., Nilsson, M., Balboni, 
T. A., Wright, A. A., Paulk, M. E. ... Prigerson, H. G. (2009). Religious coping and use of intensive life-prolonging care near death in patients with advanced cancer. JAMA, 301(11), 1140. doi: 10.1001/ jama.2009.341

Piedmont, R. L. (2012). Overview and development of measure of numinous constructs: The Assessment of Spirituality and Religious Sentiments (ASPIRES) Scale. En L. J. Miller (Ed.), The Oxford Handbook of Psychology and Spirituality (pp. 104122). Oxford: Oxford University Press.

Pirutinsky, S., Rosmarin, D. H., Pargament, K. I., \& Midlarsky, E. (2011). Does negative religious coping accompany, precede, or follow depression among Orthodox Jews? Journal of Affective Disorders, 132(3), 401-405. doi: 10.1016/j.jad.2011.03.015

Ramirez, S. P., Macêdo, D. S., Sales, P. M. G., Figueiredo, S. M., Daher, E. F., Araújo, S. M. ... Carvalho, A. F. (2012). The relationship between religious coping, psychological distress and quality of life in hemodialysis patients. Journal of Psychosomatic Research, 72(2), 129-135. doi: 10.1016/j.jpsychores.2011.11.012

Rand, K. L., Cripe, L. D., Monahan, P. O., Tong, Y., Schmidt, K., \& Rawl, S. M. (2012). Illness appraisal, religious coping, and psychological responses in men with advanced cancer. Supportive Care in Cancer, 20(8), 1719-1728. doi: 10.1007/s00520011-1265-y

Rivera-Ledesma, A., \& Montero-López, L. M. (2007). Medidas de afrontamiento religioso y espiritualidad en adultos mayores mexicanos. Salud Mental, 30(1), 39-46. Recuperado de: http://new.medigraphic.com/cgi-bin/publicaciones.cgi?IDREVIS$\mathrm{TA}=81$

Roberts, L. R., \& Montgomery, S. B. (2015). Mindfulness-based intervention for perinatal grief after stillbirth in rural India. Issues in Mental Health Nursing, 36(3), 222-230. doi: 10.3109/01612840.2014.962676

Robles-García, R., López-Luna, S., Páez, F., Escamilla, R., Camarena, B., \& Fresán, A. (2014). History of religious delusions and psychosocial functioning among Mexican patients with paranoid schizophre- nia. Journal of Religion and Health, 53(6), 16221633. doi: 10.1007/s10943-013-9727-9

Rodríguez-Carrión, D. M., Sayers-Montalvo, S., \& Martínez-Taboas, A. (2011). Contribución del manejo religioso a la calidad de vida y sintomatología depresiva en una muestra de hispanos que tienen un diagnóstico de cáncer. Revista Puertorriqueña de Psicología, 22, 27-45. Recuperado de: http://pepsic.bvsalud.org/scielo.php?script=sci_issues\&pi$\mathrm{d}=1946-2026 \& \operatorname{lng}=$ es\&nrm=iso

Rosmarin, D. H., Bigda-Peyton, J. S., Öngur, D., Pargament, K. I., \& Björgvinsson, T. (2013). Religious coping among psychotic patients: Relevance to suicidality and treatment outcomes. Psychiatry Research, 210(1), 182-187. doi: 10.1016/j.psychres.2013.03.023

Schaal, S., Heim, L., \& Elbert, T. (2014). Posttraumatic stress disorder and appetitive aggression in Rwandan genocide perpetrators. Journal of Aggression, Maltreatment \& Trauma, 23(9), 930-945. doi: 10.1080/10926771.2014.956916

Schreiber, J. B., Nora, A., Stage, F. K., Barlow, E. A., \& King, J. (2006). Reporting structural equation modeling and confirmatory factor analysis results: A review. The Journal of Educational Research, 99(6), 323-338. doi: 10.3200/JOER.99.6.323-338

Scientific Software International (2006). LISREL (8). Recuperado de http://www.ssicentral.com/lisrel

Thuné-Boyle, I. C. V., Stygall, J., Keshtgar, M. R. S., Davidson, T. I., \& Newman, S. P. (2013). Religious/ spiritual coping resources and their relationship with adjustment in patients newly diagnosed with breast cancer in the UK. Psycho-Oncology, 22(3), 646-658. doi: 10.1002/pon.3048

Trevino, K. M., Archambault, E., Schuster, J., Richardson, P., \& Moye, J. (2012). Religious coping and psychological distress in military veteran cancer survivors. Journal of Religion and Health, 51(1), 87-98. doi: 10.1007/s10943-011-9526-0

Vallurupalli, M., Lauderdale, K., Balboni, M. J., Phelps, A. C., Block, S. D., Ng, A. K. ... Balboni, T. A. (2012). The role of spirituality and religious coping in the quality of life of patients with advanced cancer receiving palliative radiation therapy. The 
Journal of Supportive Oncology, 10(2), 81-87. doi:

10.1016/j.suponc.2011.09.003

Watt, M. H., Wilson, S. M., Joseph, M., Masenga, G., Mac-

Farlane, J. C., Oneko, O., \& Sikkema, K. J. (2014).

Religious coping among women with obstetric fistula in Tanzania. Global Public Health, 9(5), 516-

527. doi: 10.1080/17441692.2014.903988

Zwingmann, C., Müller, C., Krber, J., \& Murken, S.

(2008). Religious commitment, religious coping and anxiety: A study in German patients with breast cancer. European Journal of Cancer Care, 17(4), 361-370. doi: 10.1111/j.1365-2354.2007.00867.x

Zwingmann, C., Wirtz, M., Müller, C., Körber, J., \& Murken, S. (2006). Positive and negative religious coping in German breast cancer patients. Journal of Behavioral Medicine, 29(6), 533-547. doi: 10.1007/s10865-006-9074-3

\section{Apéndice I}

Ítems de la Escala Abreviada de Afrontamiento Religioso adaptada al contexto argentino.

\begin{tabular}{|c|c|c|c|c|c|}
\hline & Nunca & $\begin{array}{c}\text { Casi } \\
\text { nunca }\end{array}$ & A veces & $\begin{array}{c}\text { Casi } \\
\text { siempre }\end{array}$ & Siempre \\
\hline Supongo que mi Iglesia por momentos me abandona. & 1 & 2 & 3 & 4 & 5 \\
\hline Trato de comprender que Dios me fortalece a través de ciertas situaciones. & 1 & 2 & 3 & 4 & 5 \\
\hline Pongo en entredicho el poder de Dios. & 1 & 2 & 3 & 4 & 5 \\
\hline Junto con Dios, intento llevar a cabo mis planes. & 1 & 2 & 3 & 4 & 5 \\
\hline Busco la ayuda de Dios, para olvidar mi enojo. & 1 & 2 & 3 & 4 & 5 \\
\hline Busco el amor y el cuidado de Dios. & 1 & 2 & 3 & 4 & 5 \\
\hline Pido perdón por mis faltas. & 1 & 2 & 3 & 4 & 5 \\
\hline Me siento castigado por Dios por mi falta de devoción. & 1 & 2 & 3 & 4 & 5 \\
\hline Pongo en duda el amor que Dios siente por mí. & 1 & 2 & 3 & 4 & 5 \\
\hline 0. Me enfoco en mi religión para dejar de preocuparme por los problemas. & 1 & 2 & 3 & 4 & 5 \\
\hline 1. Me pregunto qué pude haber hecho para que Dios me castigue de esa manera. & 1 & 2 & 3 & 4 & 5 \\
\hline 12. Supongo que Dios me abandona. & 1 & 2 & 3 & 4 & 5 \\
\hline 13. Me siento convencido que el demonio hace que las cosas pasen. & 1 & 2 & 3 & 4 & 5 \\
\hline 14. Busco la cercanía de Dios. & 1 & 2 & 3 & 4 & 5 \\
\hline
\end{tabular}

